INPLASY

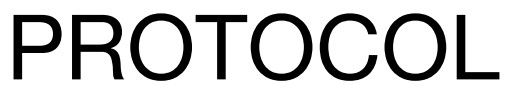

To cite: Ren et al. The effects of traditional Chinese manual therapy (Tuina) for chronic fatigue syndrome: a protocol for systematic review and meta-analysis. Inplasy protocol 2021100025. doi:

10.37766/inplasy2021.10.0025

Received: 07 October 2021

Published: 08 October 2021

Corresponding author:

Kong LingJun

chunyong01@163.com

Author Affiliation:

Yueyang Hospital of Integrated

Traditional Chinese and

Western Medicine, Shanghai

University of Traditional

Chinese Medicine.

Support: Scientific research projects.

Review Stage at time of this submission: Preliminary

searches.

Conflicts of interest:

None declared.

\section{The effects of traditional Chinese manual therapy (Tuina) for chronic fatigue syndrome: a protocol for systematic review and meta-analysis}

\author{
Ren, J1; He, T2; Zhou, X33 Wu, Z4; Kong, L5.
}

Review question / Objective: This systematic review and meta-analysis will provide a relatively convincing conclusion as to whether Tuina can effectively treat fatigue symptoms in patients with CFS.

Condition being studied: The effects of traditional Chinese manual therapy (Tuina) for chronic fatigue syndrome. Papers are screened through websites such as PubMed, and then systematic review and meta-analysis of the articles that meet the requirements will be carried out.

Information sources: The comprehensive electronic search of PubMed, Web of Science, Chinese National Knowledge Infrastructure, Wanfang Database, Embase, Cochrane Library, Chinese Science Citation Database, Technology Periodical Database from their inception to October 2021 will be conducted.

INPLASY registration number: This protocol was registered with the International Platform of Registered Systematic Review and Meta-Analysis Protocols (INPLASY) on 08 October 2021 and was last updated on 08 October 2021 (registration number INPLASY2021100025).

\section{INTRODUCTION}

Review question / Objective: This systematic review and meta-analysis will provide a relatively convincing conclusion as to whether Tuina can effectively treat fatigue symptoms in patients with CFS.
Condition being studied: The effects of traditional Chinese manual therapy (Tuina) for chronic fatigue syndrome. Papers are screened through websites such as PubMed, and then systematic review and 
meta-analysis of the articles that meet the requirements will be carried out.

\section{METHODS}

Participant or population: Participants diagnosed as CFS.

Intervention: In treatment group, the patients with CFS were treated by Tuina therapy.

Comparator: In control group, the patients received medicine, observation, education, sham manual therapy, and so on.

Study designs to be included: Randomized controlled trials of Tuina therapy for chronic fatigue syndrome will be included.

Eligibility criteria: Participants diagnosed as CFS met the diagnostic criteria of CFS revised by the Centers for Disease Control and Prevention in 1994, International Consensus Criteria for ME.

Information sources: The comprehensive electronic search of PubMed, Web of Science, Chinese National Knowledge Infrastructure, Wanfang Database, Embase, Cochrane Library, Chinese Science Citation Database, Technology Periodical Database from their inception to October 2021 will be conducted.

Main outcome(s): The primary outcome is assessed by the following fatigue outcomes scales: Fatigue Rating Scale, Fatigue Scale-14, Brief Fatigue Inventory, Fatigue Severity Scale, and Fatigue Impact Scale.

Quality assessment / Risk of bias analysis: Quality of evidence will be assessed using the Grades of Recommendation, Assessment, Development and Evaluation. The result of the assessment will be crosschecked.

Strategy of data synthesis: The metaanalysis will be conducted using the Review Manager Version 5.3 software. The heterogeneity will be assessed using the $I^{2}$ statistic and $Q$ statistic.

Subgroup analysis: The subgroup analysis will be conducted based on the duration of treatment, age, gender, duration of CFS.

Sensitivity analysis: Sensitivity Analysis will be used to evaluate the reliability of the synthesis results of the meta-analysis of each result index.

Country(ies) involved: China.

Keywords: Tuina, chronic fatigue syndrome, fatigue.

Contributions of each author:

Author 1 - Ren Jun - The author drafted the manuscript, and work out the research strategy, and contributed to the development of conceptualization.

Author 2 - He Tianxiang - The author drafted the manuscript, and work out the research strategy. And the author provided the funding, contributed to the development of conceptualization.

Author 3 - Zhou Xin - drafted the manuscript, work out the research strategy, and contributed to the development of the project administration.

Author 4 - Wu Zhiwei - The author contributed to the development of the project administration, and read, provided feedback and approved the final manuscript.

Author 5 - Kong Lingjun - The author read, provided feedback and approved the final manuscript, and contributed to the development of conceptualization, funding acquisition. 